S. TANNO

KODAI MATH. J.

5 (1982), 230-237

\title{
A CHARACTERIZATION OF A COMPLEX PROJECTIVE SPACE BY THE SPECTRUM
}

\author{
By SHUKICHI TANNO
}

\section{Introduction}

Let $(M, g)$ be a compact $m$-dimensional Riemannian manifold and $\left(S^{m}, g_{0}\right)$ be an $m$-sphere of constant curvature 1 . We denote the spectrum of the Laplacian acting on functions on $(M, g)$ by $\operatorname{Spec}(M, g)$. If $m \leqq 6$, $\operatorname{Spec}(M, g)=\operatorname{Spec}\left(S^{m}, g_{0}\right)$ implies that $(M, g)$ is isometric to $\left(S^{m}, g_{0}\right)$ (Berger [1], Tanno [8]). For $m \geqq 7$ it is an open question if $\left(S^{m}, g_{0}\right)$ is characterized by the spectrum. In [9] we proved the following.

Theorem A (Tanno [9]). Assume that $\operatorname{Spec}(M, g)=\operatorname{Spec}\left(S^{m}, g_{0}\right)$. If $g$ is sufficiently close to constant curvature metric, then $(M, g)$ is isometric to $\left(S^{m}, g_{0}\right)$.

In this paper we give the Kähler version of Theorem A. Let $(M, J, g)$ be a compact Kählerian manifold of dimension $m=2 n$, and $\left(C P^{n}, J_{0}, g_{0}\right)$ be a complex projective space with the Fubini-Study metric of constant holomorphic sectional curvature 4 . Then, we get the following. (Cf. Proposition 2.1.)

Theorem B. Assume that $\operatorname{Spec}(M, J, g)=\operatorname{Spec}\left(C P^{n}, J_{0}, g_{0}\right)$. If $g$ is sufficiently close to constant holomorphic sectional curvature metric, then $(M, J, g)$ is holomorphically isometruc to $\left(C P^{n}, J_{0}, g_{0}\right)$.

As for isospectral deformations of flat metrics, see [4], [5], and for inverse spectral results for negatively curved manifolds, see [2], [3].

\section{Preliminaries}

Let $(M, J, g)$ be a Kählerian manifold, where $J=\left(J_{j}^{i}\right)$ denotes the complex structure tensor and $g=\left(g_{\imath j}\right)$ a Kähler metric. By $R=\left(R_{j k l}^{\imath}\right), \rho=\left(R_{j l}\right)$ and $S$ we denote the Riemannian curvature tensor, the Ricci curvature tensor and the scalar curvature of $(M, J, g)$, respectively. We put

$$
R_{\imath \jmath}^{*}=R_{\imath r} J_{\jmath}^{r} .
$$

Received December 5, 1980 
Then we have the following classical relations:

$$
\begin{array}{ll}
2 R_{\imath \jmath}^{*}=R_{\imath \jmath r s} J^{r s}=2 R_{\imath r \jmath s} J^{r s}, \\
R_{\imath \jmath k l}=J_{\imath}^{r} J_{\jmath}^{s} R_{r s k l}, & J_{r}^{i} R_{\jmath k l}^{r}=J_{j}^{r} R_{r k l}^{\imath}, \\
R_{r s} J_{\imath}^{r} J_{\jmath}^{s}=R_{\imath \jmath}, & R_{r s}^{*} J_{\imath}^{r} J_{\jmath}^{s}=R_{\imath \jmath}^{*}, \\
R_{\imath \jmath}^{*} J^{i \jmath}=S, & R_{\imath \jmath}^{*} R^{* \imath \jmath}=R_{\imath j} R^{\imath \jmath} .
\end{array}
$$

The Bochner curvature tensor $B=\left(B_{j k l}^{\imath}\right)$ is by definition

$$
\begin{aligned}
B_{\imath j k l}= & R_{\imath j k l}-\left(g_{i k} R_{\jmath l}-g_{i l} R_{j k}+R_{\imath k} g_{j l}-R_{i l} g_{\jmath k}+J_{\imath k} R_{j l}^{*}-J_{i l} R_{j k}^{*}\right. \\
& \left.+R_{i k}^{*} J_{j l}-R_{i l}^{*} J_{j k}+2 R_{\imath \jmath}^{*} J_{k l}+2 J_{\imath j} R_{k l}^{*}\right) /(m+4) \\
& +S\left(g_{\imath k} g_{j l}-g_{i l} g_{j k}+J_{\imath k} J_{j l}-J_{i l} J_{j k}+2 J_{\imath \jmath} J_{k l}\right) /(m+2)(m+4),
\end{aligned}
$$

where $m=2 n=\operatorname{dim} M$. Easily we get

$$
\begin{aligned}
& g^{\imath k} B_{\imath j k l}=0, \quad J^{k l} B_{\imath j k l}=0, \\
& J^{i k} B_{\imath j k l}=0 .
\end{aligned}
$$

We use the following notations:

$$
\begin{aligned}
& (P, Q)=P_{\imath \jmath k l} Q^{\imath j k l}, \quad|P|^{2}=(P, P), \\
& (P, Q, T)=P^{\imath \jmath}{ }_{k l} Q_{r s}^{k l} T^{r s}{ }_{\imath \jmath}, \\
& (U ; Q, T)=U^{r s} Q_{r j k l} T_{s}{ }^{j k l}, \\
& (U ; V ; T)=U^{i k} V^{j l} T_{\imath j k l}, \\
& (U V W)=U^{i}{ }_{j} V_{k}^{\jmath} W^{k}{ }_{\imath},
\end{aligned}
$$

where $P, Q$, and $T$ are tensor fields of type $(1,3)$; and $U, V$, and $W$ are tensor fields of type $(1,1)$. obtain

In the following calculations the methods are similar to ones in [9]. We

$$
\begin{aligned}
(R, R, R)= & (R, B, B)+8(\rho ; R, B) /(n+2)+8(\rho ; \rho ; B) /(n+2) \\
& -2 S(R, B) /(n+1)(n+2)+24(\rho \rho \rho) /(n+2)^{2} \\
& +4(n+6)(\rho ; \rho ; R) /(n+2)^{2}+2(n-9) S(\rho, \rho) /(n+1)(n+2)^{2} \\
& -(n-1) S^{3} /(n+1)^{2}(n+2)^{2}, \\
& (\rho ; R, B)=(\rho ; B, B)+4(\rho ; \rho ; B) /(n+2),
\end{aligned}
$$




$$
\begin{aligned}
(\rho ; R, R)= & (\rho ; R, B)+4[(\rho \rho \rho)+(\rho ; \rho ; R)] /(n+2) \\
& -2 S(\rho, \rho) /(n+1)(n+2), \\
& (R, B)=(B, B), \\
(\rho ; \rho ; R)= & (\rho ; \rho ; B)+(2 n+1) S(\rho, \rho) / 2(n+1)(n+2) \\
& +2(\rho \rho \rho) /(n+2)-S^{3} / 4(n+1)(n+2),
\end{aligned}
$$

where we have used the following

$$
B_{\imath j k l} R^{* \imath \jmath} R^{* k l}=2(\rho ; \rho ; B)
$$

etc.

Next we assume that $M$ is compact. Let $\lambda_{2}$ be the $\imath$-th eigenvalue of the Laplacian acting on functions on $(M, g)$ and let $\operatorname{Spec}(M, g)$ denote the spectrum of $(M, g)$. Then the asymptotic expansion by Minakshisundaram-Pleijel is

$$
\sum e^{-\lambda_{i} t} \sim(4 \pi t)^{-n} \sum_{\beta=0} a_{\beta} t^{\beta} \quad(t \downarrow 0),
$$

where $a_{\beta}=a_{\beta}(M, g)$ and

$$
\begin{gathered}
a_{0}=\operatorname{Vol}(M, g), \\
a_{1}=(1 / 6) \int_{M} S, \\
a_{2}=(1 / 360) \int_{M}\left[2|R|^{2}-2|\rho|^{2}+5 S^{2}\right], \\
a_{3}=(1 / 6 !) \int_{M}\left[-Z+2 S|R|^{2} / 3-2 S|\rho|^{2} / 3+5 S^{3} / 9+A\right],
\end{gathered}
$$

where we have put

$$
\begin{aligned}
& Z=|\nabla R|^{2} / 9+26|\nabla \rho|^{2} / 63+142|\nabla S|^{2} / 63, \\
& A=8(R, R, R) / 21-8(\rho ; R, R) / 63+20(\rho ; \rho ; R) / 63-4(\rho \rho \rho) / 7 .
\end{aligned}
$$

We put

Then, $(g, G)=0$ and

$$
G_{j l}=R_{j l}-S g_{j l} / 2 n .
$$

$$
\begin{gathered}
|G|^{2}=|\rho|^{2}-S^{2} / 2 n, \\
(\rho ; \rho ; B)=(G ; G ; B), \\
(\rho \rho \rho)=(\rho G G)+S|G|^{2} / n+S^{3} / 4 n^{2} .
\end{gathered}
$$

Since

$$
|B|^{2}=|R|^{2}-8|\rho|^{2} /(n+2)+2 S^{2} /(n+1)(n+2),
$$


we obtain (cf. [8])

$$
2|R|^{2}-2|\rho|^{2}+5 S^{2}=2|B|^{2}+2(6-n)|G|^{2} /(n+2)
$$

$$
+\left(5 n^{2}+4 n+3\right) S^{2} / n(n+1) \text {. }
$$

By (1.1) (1.5), we obtain

where

$$
\begin{aligned}
A= & 8(R, B, B) / 21+8(22-n)(\rho ; B, B) / 63(n+2) \\
& -16 S|B|^{2} / 21(n+1)(n+2)+p \cdot(\rho ; \rho ; B) \\
& +q \cdot(\rho \rho \rho)+u S|\rho|^{2}+v S^{3},
\end{aligned}
$$

$$
\begin{gathered}
p=4\left(5 n^{2}+76 n+420\right) / 63(n+2)^{2}, \\
q=8\left(n^{2}+92 n+276\right) / 63(n+2)^{3}-4 / 7 \\
=-4\left(9 n^{3}+52 n^{2}-76 n-480\right) / 63(n+2)^{3}, \\
u=2\left(10 n^{3}+109 n^{2}+196 n-252\right) / 63(n+1)(n+2)^{3}, \\
v=-\left(5 n^{3}+65 n^{2}+208 n+100\right) / 63(n+1)^{2}(n+2)^{3} .
\end{gathered}
$$

LEMMA 1.1. $a_{3}$ and $A$ are expressed as follows:

$$
\begin{aligned}
a_{3}= & (1 / 6 !) \int_{M}\left[-Z+2 S|B|^{2} / 3+2(6-n) S|G|^{2} / 3(n+2)\right. \\
& \left.+(5 / 9-(n-3) / 3 n(n+1)) S^{3}+A\right], \\
A= & 8(R, B, B) / 21+8(22-n)(\rho ; B, B) / 63(n+2) \\
& -16 S|B|^{2} / 21(n+1)(n+2)+p \cdot(G ; G ; B) \\
& +q \cdot(\rho G G)+(q / n+u) S|G|^{2}+\left(q / 4 n^{2}+u / 2 n+v\right) S^{3} .
\end{aligned}
$$

Proof. Eliminating $S\left(|R|^{2}-|\rho|^{2}\right)$ from (1.9) by (1.13) we get (1.19). By (1.10) (1.12) and (1.14) we obtain (1.20).

Q.E.D.

A Kählerian manifold $(M, J, g)$ is of constant holomorphic sectional curvature 4 , if and only if

$$
R_{\imath j k l}=g_{\imath k} g_{j l}-g_{i l} g_{j k}+J_{\imath k} J_{j l}-J_{i l} J_{j k}+2 J_{\imath \jmath} J_{k l} .
$$

We define $E$ as the space of all (0,4)-tensor fields $T$ satisfying the following conditions ;

$$
\begin{array}{ll}
T_{\imath j k l}=T_{k \imath \imath \jmath}, & T_{\imath j k l}=-T_{j i k l}, \\
T_{\imath j k l} J_{r}^{i} J_{s}^{j}=T_{r s k l}, & J^{i j} T_{\imath j k l}=0 .
\end{array}
$$

The Bochner curvature tensor $B$ belongs to $E$. 
If $(M, J, g)$ is of constant holomorphic sectional curvature 4 , then we obtain

$$
(R, T, T)=4(T, T)
$$

for any $T$ in $E$. This follows from (1.21) and the definition of $E$.

A Kählerian manifold $(M, J, g)$ is of constant holomorphic sectional curvature, if and only if $B=G=0$.

\section{Kähler metrics close to constant holomorphic sectional curvature metric}

Let $\left(C P^{n}, J_{0}, g_{0}\right)$ be a complex projective space with the Fubini-Study metric of constant holomorphic sectional curvature 4 . Then the scalar curvature $S_{0}$ is equal to $4 n(n+1)$. We put $a_{\beta}^{0}=a_{\beta}\left(C P^{n}, J_{0}, g_{0}\right)$

PROPOSITION 2.1. There exists a positzve number $\delta=\delta(n)<1$ with the following property: Assume that a compact Kählerian manifold $(M, J, g), \operatorname{dim} M=2 n$, satısfies the following conditions;

(i) $(R, T, T) \leqq 4(1+\delta)(T, T) \quad$ for $T \in E$,

(ii) $2(n+1)(1-\delta)<$ Riccl curvature $<2(n+1)(1+\delta)$,

(iii) $|B|<\delta$,

(iv) $a_{\beta}=a_{\beta}^{0}, \quad \beta=0,1,2,3$.

Then $(M, J, g)$ is holomorphically isometric to $\left(C P^{n}, J_{0}, g_{0}\right)$.

If $n \leqq 6, \operatorname{Spec}(M, J, g)=\operatorname{Spec}\left(C P^{n}, J_{0}, g_{0}\right)$ implies that $(M, J, g)$ is holomorphically isometric to $\left(C P^{n}, J_{0}, g_{0}\right)$ (cf. [8]). Therefore in the proof of the Proposition 2.1 we can assume that $n \geqq 7$. We assume that $(M, J, g)$ satisfies (i) (iv) and show that $\delta$ can be determined so that $(M, J, g)$ is holomorphically isometric to $\left(C P^{n}, J_{0}, g_{0}\right) . \quad a_{0}=a_{0}^{0}$ means that $\operatorname{Vol}(M, J, g)=\operatorname{Vol}\left(C P^{n}, J_{0}, g_{0}\right)$. $a_{1}=a_{1}^{0}$ implies that $\int S=4 n(n+1) \operatorname{Vol}\left(C P^{n}, J_{0}, g_{0}\right)$.

LEMMA 2.2. We have $S>0$ and

$$
\begin{gathered}
|S-4 n(n+1)|<4 n(n+1) \delta \\
\int_{M}\left[S^{2}-S_{0}^{2}\right] \geqq 0 \\
0 \leqq \int_{M}\left[S^{3}-S_{0}^{3}\right] \leqq(3+\delta) 4 n(n+1) \int_{M}\left[S^{2}-S_{0}^{2}\right] .
\end{gathered}
$$

Proof. $\quad S>0$ and (2.1) follow from (ii). As for (2.2) and (2.3) see Lemma 3 and Lemma 6 in [9].

Q.E.D. 
By (1.13) and $a_{2}=a_{2}^{0}$ we get

$$
\begin{aligned}
2(n-6) \int_{M}|G|^{2}= & 2(n+2) \int_{M}|B|^{2} \\
& +\left[(n+2)\left(5 n^{2}+4 n+3\right) / n(n+1)\right] \int_{M}\left[S^{2}-S_{0}^{2}\right]
\end{aligned}
$$

By Lemma 1.1 and $a_{3}=a_{3}^{0}$ we obtain

$$
\begin{aligned}
D:=\int_{M}[ & -Z+8(R, B, B) / 21+8(22-n)(\rho ; B, B) / 63(n+2) \\
& +[2 / 3-16 / 21(n+1)(n+2)] S|B|^{2}+p \cdot(G ; G ; B) \\
& +q \cdot(\rho G G)+[q / n+u-2(n-6) / 3(n+2)] S|G|^{2} \\
& +\left[q / 4 n^{2}+u / 2 n+v+5 / 9-(n-3) / 3 n(n+1)\right]\left(S^{3}-S_{0}^{3}\right) \\
=0 . &
\end{aligned}
$$

LeMma 2.3. We put $\mu=1$ if $22>n$ and $\mu=-1$ if $22<n$. Then

$$
\begin{gathered}
(22-n)(\rho ; B, B) \leqq(22-n) 2(n+1)(1+\mu \delta)|B|^{2}, \\
2 / 3-16 / 21(n+1)(n+2)>0, \quad p>0, \quad v<0, \\
q / n+u-2(n-6) / 3(n+2)<0, \\
q / 4 n^{2}+u / 2 n+v+5 / 9-(n-3) / 3 n(n+1)>0, \\
q \cdot(\rho G G) \leqq 2 q(n+1)(1-\delta)|G|^{2}, \\
(G ; G ; B) \leqq \delta|G|^{2} .
\end{gathered}
$$

Proof. (2.5) follows from (ii). (2.6) is trivial. To prove (2.8) first we get

$$
q / 4 n^{2}+u / 2 n+v=-4\left(n^{2}-2 n-15\right) / 63 n^{2}(n+1)^{2} .
$$

Then (2.8) is clear. (2.9) follows from (ii). (2.10) follows from (iii) (cf. Lemma 7 in [9]).

Applying (i), (ii), Lemma 2.2 and Lemma 2.3 to $D$ we get Q.E.D.

$$
\begin{aligned}
D \leqq \int_{M}[ & -Z+32(1+\delta)|B|^{2} / 21 \\
& +16(22-n)(n+1)(1+\mu \delta)|B|^{2} / 63(n+2) \\
& +[2 / 3-16 / 21(n+1)(n+2)] 4 n(n+1)(1+\delta)|B|^{2} \\
& +p \delta|G|^{2}+2(n+1)(1-\delta) q|G|^{2} \\
& +[q / n+u-2(n-6) / 3(n+2)] 4 n(n+1)(1-\delta)|G|^{2} \\
& \left.+\left[q / 4 n^{2}+u / 2 n+v+5 / 9-(n-3) / 3 n(n+1)\right](3+\delta) 4 n(n+1)\left(S^{2}-S_{0}^{2}\right)\right] .
\end{aligned}
$$


Next applying (2.4) to $|G|^{2}$ in (2.12) we obtain

$$
D \leqq \int_{M}\left[-Z-(U-P \delta)|B|^{2}-(V-Q \delta)\left(S^{2}-S_{0}^{2}\right)\right],
$$

where we have put

$$
\begin{aligned}
U= & -32 / 21-16(22-n)(n+1) / 63(n+2)+64 n / 21(n+2) \\
& -2(n+1)(n+2)(3 q+2 n u) /(n-6), \\
P= & 32 / 21+16(22-n)(n+1) \mu / 63(n+2) \\
& +[4 / 3-16 / 21(n+1)(n+2)] 4 n(n+1) \\
& +(n+1)(n+2)(p /(n+1)-6 q-4 n u) /(n-6), \\
V= & -12 n(n+1)\left[q / 4 n^{2}+u / 2 n+v+5 / 9-(n-3) / 3 n(n+1)\right] \\
& -(n+2)\left(5 n^{2}+4 n+3\right)[3 q+2 n u-4 n(n-6) / 3(n+2)] / n(n-6), \\
Q= & 4 n(n+1)\left[q / 4 n^{2}+u / 2 n+v+5 / 9-(n-3) / 3 n(n+1)\right] \\
& +(n+2)\left(5 n^{2}+4 n+3\right)[p /(n+1)-6 q-4 n u+8 n(n-6) / 3(n+2)] / 2 n(n-6) .
\end{aligned}
$$

By calculations we get

$$
\begin{gathered}
3 q+2 n u=-\left(68 n^{2}+24 n-1440\right) / 63(n+1)(n+2), \\
U=2\left(76 n^{4}+144 n^{3}-960 n^{2}-3136 n-2496\right) / 63(n+2)^{2}(n-6) .
\end{gathered}
$$

Therefore we see that $U$ is positive. Since $q / 4 n^{2}+u / 2 n+v$ is negative by (2.11) and $3 q+2 n u$ is negative by (2.14), we get

$$
\begin{aligned}
V & >-12 n(n+1)[5 / 9-(n-3) / 3 n(n+1)]+4\left(5 n^{2}+4 n+3\right) / 3 \\
& =8(n-3) / 3 .
\end{aligned}
$$

Therefore $V$ is also positive. Since $P$ and $Q$ are positive, we can define $\delta$ by

$$
\delta=\min (U / P, V / Q, 99 / 100) \text {. }
$$

Proof of Proposition 2.1. By the definition of $\delta$, (2.13) shows that $Z=B=0$ and $S=S_{0}$. Furthermore (2.4) shows that $G=0$, and hence $(M, J, g)$ is of constant holomorphic sectional curvature 4 . So $(M, J, g)$ is holomorphically isometric to $\left(C P^{n}, J_{0}, g_{0}\right)$.

Q.E.D.

\section{REFERENCES}

[1] M. Berger, Le spectre des variétés riemanniennes, Rev. Roum. Math. Pure Appl., 13 (1969), 915-931. 
[2] V. Guillemin and D. Kazhdan, Some inverse spectral results for negatively curved 2-manifolds, Topology, 19 (1980), 301-312.

[3] V. Guillemin And D. Kazhdan, Some inverse spectral results for negatively curved $n$-manifolds, Proc. Symp. Pure Math. 36 (1980), 135-180.

[4] R. Kuwabara, On isospectral deformations of Riemannian metrics, Compositio Math., 40 (1980), 319-324.

[5] R. Kuwabara, On the characterization of flat metrics by the spectrum, to appear.

[6] H.F. Mckean And I. M. Singer, Curvature and the eigenvalues of the Laplacian, Journ. Diff. Geom., 1 (1967), 43-69.

[7] T. SAKAI, On eigenvalues of Laplacian and curvature of Riemannian manifold, Tôhoku Math. Journ., 23 (1971), 589-603.

[8] S. TANno, Eigenvalues of the Laplacian of Riemannian manifolds, Tôhoku Math. Journ., 25 (1973), 391-403.

[9] S. TAnno, A characterization of the canonical spheres by the spectrum, Math. Zeit., 175 (1980), 267-274.

Department of Mathematics

Tokyo Institute of Technology 